\title{
ESO large program on physical studies of Transneptunian Objects and Centaurs: Visible photometry - First results ${ }^{\star}$
}

\author{
H. Boehnhardt ${ }^{1}$, A. Delsanti ${ }^{1,2}$, A. Barucci ${ }^{2}$, O. Hainaut ${ }^{1}$, A. Doressoundiram ${ }^{2}$, M. Lazzarin ${ }^{3}$, L. Barrera ${ }^{4}$ \\ C. de Bergh ${ }^{2}$, K. Birkle ${ }^{5}$, E. Dotto ${ }^{6,7}$, K. Meech ${ }^{8}$, J. E. Ortiz ${ }^{9}$, J. Romon ${ }^{2}$, T. Sekiguchi ${ }^{10}$, N. Thomas ${ }^{11}$, \\ G. P. Tozzi ${ }^{12}$, J. Watanabe ${ }^{10}$, and R. M. West ${ }^{13}$
}

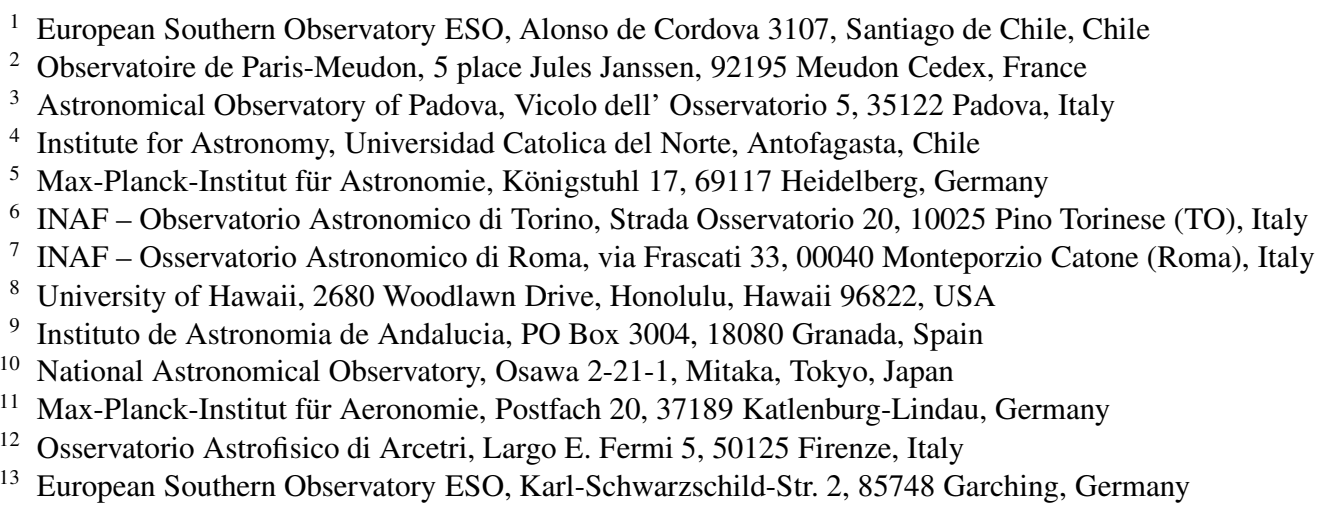

Received 7 May 2002 / Accepted 30 August 2002

\begin{abstract}
We present the first results of BVRI photometry of Transneptunian Objects (TNOs) and Centaurs obtained through the ESO Large Program on physical studies of these icy bodies in the outer solar system. In total 28 objects were observed of which 18 are new measurements. Combining our new BVRI photometry with the data summary published by Hainaut \& Delsanti (2002) results in a database of 94 objects: 45 Cubewanos, 22 Plutinos, 13 scattered disk objects, 14 Centaurs. The reddening range seems to be similar among the four dynamical classes (-5 to 55\%/100 nm) and only one outlier $\left(1994 \mathrm{ES}_{2}\right)$ exists. The spectral gradient distribution of the Cubewanos peaks between 25 to 35\%/100 nm, while for the three other types the maximum seems to fall below 20\%/100 $\mathrm{nm}$. A clustering of red Cubewanos with perihelia beyond $\sim 41$ AU in low eccentricity and low inclination orbit suggests that these objects are less affected by the physical processes that potentially produce neutral colors, i.e. resurfacing by collision and by intrinsic activity. For Cubewanos and scattered disk objects, the range of reddening increases with decreasing perihelion distance and with increasing orbital excitation. A correlation of the spectral slope with inclination is present for Cubewanos and scattered disk objects, and is non-existent for the other dynamical types. It is unclear whether these trends (or their absence) are discriminative for the correctness of the resurfacing scenarios. If intrinsic activity is responsible for resurfacing, the start of the effect inside $\sim 41 \mathrm{AU}$ from the Sun may be indicative for the driving agent, while in the collision scenario the survival of the red Cubewano cluster in the central region of the Kuiper-Belt argues for the existence of a population of bodies the surface of which is heavily radiation processed without impact resurfacing.
\end{abstract}

Key words. Kuiper-Belt - minor planets, asteroids - techniques: photometric

\section{Introduction}

The Kuiper-Belt region is the origin of icy bodies that are among the most pristine solar system objects observable from Earth: Transneptunian Objects (TNOs) and Centaurs. Among TNOs three dynamical classes are identified: the Plutinos with orbits in the $2 / 3$ resonance to Neptune (like

Send offprint requests to: $\mathrm{H}$. Boehnhardt,

e-mail: hboehnha@eso.org

* Based on observations collected at the European Southern Observatory, Chile, program 167.C-0340.
Pluto/Charon), the Cubewanos in slightly elliptical orbits with semi-major axes between about 40 and $46 \mathrm{AU}$ and the scattered disk objects in highly eccentric orbits and with semi-major axes of at least the distance of Cubewanos. The Centaurs, which are probably escapees from the Kuiper-Belt, appear to be the 4th dynamical class of TNOs with eccentric orbits between the distance of Jupiter and Neptune. For a description of the dynamical classes of TNOs and their evolution see Levison (2002).

Physical studies of TNOs and Centaurs are based on visible and near-IR photometry and spectroscopy addressing global colors and spectral gradients as well as spectral absorption 
features that are characteristic of the surface material of the objects. By far, the most numerous data on TNOs and Centaurs (more than 60 TNOs and 15 Centaurs) are available in the form of broad band $B V R I$ photometry, much less (in total 7 TNOs and 6 Centaurs) with $J H K$ photometry; see Hainaut \& Delsanti (2002) for a listing of visible and near-IR colors plus references to the original papers.

After some precursor programs at ESO telescopes a consortium of scientists (see list of authors) has proposed a comprehensive observing and analysis project on physical properties of TNOs and Centaurs, to be performed within the framework of an ESO Large Program. The project was accepted to be executed at ESO telescopes in Cerro Paranal and La Silla during April 2001 to March 2003. The main goals and the implementation of the project are described in Boehnhardt et al. (2002).

In this paper we present and discuss the observations of the first set of 28 objects, measured photometrically through $B V R I$ filters during the first semester of the project. Further results - visible and near-IR photometry as well as spectroscopy - will be published elsewhere.

\section{Observations at the ESO VLT}

The CCD photometry of TNOs and Centaurs was performed between April and August 2001 at the Very Large Telescope (VLT) facility of the European Southern Observatory ESO in Chile, in both visitor and service mode. During this period 24 hours of service mode time were scheduled for our program. The service mode exposures were performed under clear to photometric conditions with dark sky and seeing $<0.8^{\prime \prime}$. Of the first two visitor mode nights only the one on 26-27 April 2001 had useful results under clear sky conditions with seeing of $0.6-1.0^{\prime \prime}$. About 15 percent of the available time of this night was used for photometry, the rest was spent on quasi-simultaneous spectroscopy of the targets.

In both cases (visitor and service mode), the FORS1 instrument mounted to one of the VLT unit telescopes (UTs) was used, i.e. on UT1 Antu until July 2001 and on UT3 Melipal as of August 2001. FORS1 was used in imaging mode with broad band Bessell BVRI filters (Bessell 1990). See the VLT observatory web page http: //www . eso.org/paranal for technical information of the telescopes and instrument used. A standard filter exposure series $R B V I R$ was executed sequentially per object within $25-45 \mathrm{~min}$. In a few cases (fainter objects) the total integration time was extended to 60-90 min. If needed (avoidance of sky saturation) the total time per filter was split in two or more exposures. The $R$ filter exposure was repeated every 30-40 min interleaved with other filters in order to allow the monitoring of object variability. For a small number of objects more than one filter exposure series were taken. In visitor mode, differential auto-guiding of the telescope at the velocity of the moving object was applied during the observations, in service mode sidereal tracking was used (since no impacts on the photometric accuracy were expected from the small trailing of the objects during the 5-15 min exposures).

Standard star fields (Landolt 1992) - at least one field close to meridian during service mode, about 4-5 fields over a wider air-mass range in visitor mode - were taken during the TNO observing nights as well as the usual set of bias and sky flatfield exposures.

\section{Data reduction}

The data reduction was performed using the MIDAS (Munich Image \& Data Analysis System) software package of ESO.

Basic reduction: the data were bias-subtracted and divided by a flat-field frame. The bias is a median frame of a series of raw bias frames taken close in time (1-2 days) to the science exposures. The flat-field used is a median frame of a series of normalized, bias-subtracted twilight flat-field frames. At this stage, we did not correct the images for bad columns or cosmic rays. We prefer to perform blemish correction in the area of the object only if necessary. Fringes in the $I$ filter were not corrected either.

Photometric calibration: the photometric calibration was done using the MIDAS TMAG package (http://www.sc. eso.org/ ohainaut/). The stars from Landolt (1992) fields were measured using a large (i.e. 15" diameter) aperture. For those observations which were performed in service mode, between one to three standard stars fields per night were available in the framework of the instrument calibration plan. When we had only one or two standard fields, the extinction could not be computed by least square fits of the standard stars measurements, since the range of air-mass was not well sampled. Thus, the photometric calibration parameters were determined as follows:

- Zero point: computed from least square fits of the standard stars and compared to the FORS1 calibration plan value given by the VLT Observatory (see http://www . eso.org/observing/dfo/quality/FORS /qc/zeropoints). The values are in good agreement, taking into account that some shift can be introduced due to slight differences in the normalization of the flat-field frames (meaning, the FORS1 calibration plan data and our images may use different bias and flat-field frames).

- Extinction: computed when possible (i.e. good air-mass range available) or FORS1 calibration plan value (see http://www . eso.org/observing/dfo/quality/FORS /qc/zeropoints).

- Color correction: provided by the FORS1 calibration plan (see http://www.eso.org/observing/dfo/quality /FORS/qc/zeropoints).

Object photometry: the objects were measured with an aperture diameter set of up to 5 or 6 times the stellar full width at half maximum (FWHM) for each individual frame. This FWHM was evaluated on each image from several nearby, nonsaturated stars. The sky background was measured in a 10 pixel (i.e. $2^{\prime \prime}$ ) wide annulus centered on the object, at $\sim 6$ FWHM from its center. As we use these magnitudes for color studies only, the resulting colors are not affected. In case of multiple exposures per object and filter a check is performed whether the results are consistent within the error bars and, if so, the mean magnitude per filter is calculated. Only in one case, $1994 \mathrm{EV}_{3}$, a significant variability of the filter brightness (see Sect. 4) was 
Table 1. Average magnitudes, spectral gradients and colors.

\begin{tabular}{|c|c|c|c|c|c|c|c|}
\hline Object & Class $^{(1)}$ & Epoch $^{(2)}$ & $\mathrm{M} 11^{(3)} \pm \sigma$ & $\mathrm{Grt}^{(4)} \pm \sigma$ & $B-V \pm \sigma$ & $V-R \pm \sigma$ & $R-I \pm \sigma$ \\
\hline $1994 \mathrm{EV}_{3}$ & QB1 & $27 / 04 / 2001$ & $7.550 \pm 0.138$ & $37.728 \pm 7.995$ & $1.065 \pm 0.110$ & $0.670 \pm 0.149$ & $0.800 \pm 0.100$ \\
\hline $1994 \mathrm{JQ}_{1}$ & QB1 & $8 / 04 / 2001$ & $6.936 \pm 0.107$ & $24.690 \pm 5.330$ & $1.242 \pm 0.152$ & $0.586 \pm 0.086$ & $0.676 \pm 0.094$ \\
\hline $1996 \mathrm{GQ}_{21}$ & Scat & $7 / 04 / 2001$ & $798 \pm 0.051$ & $37.371 \pm 2.853$ & $1.011 \pm 0.068$ & $0.726 \pm 0.043$ & $4 \pm 0.056$ \\
\hline $1996 \mathrm{RR}_{20}$ & Plut & $15 / 08 / 2001$ & $7.575 \pm 0.103$ & $37.766 \pm 4.116$ & $1.111 \pm 0.151$ & $0.801 \pm 0.074$ & $0.551 \pm 0.055$ \\
\hline $1996 \mathrm{SZ}_{4}$ & Plut & 01 & $565 \pm 0.054$ & $9.161 \pm 3.969$ & $0.670 \pm 0.073$ & $0.492 \pm 0 .($ & $0.377 \pm 0.068$ \\
\hline $1997 \mathrm{QH}_{4}$ & QB1 & $6 / 07$ & $=0.095$ & 29.579 & +0.139 & $0.671 \pm 0.067$ & $0.619 \pm 0.050$ \\
\hline $1997 \mathrm{QJ}_{4}$ & ut & $26 / 07 / 2001$ & $047 \pm 0.062$ & $-1.507 \pm 3.558$ & $0.808 \pm$ & $0.296 \pm 0.058$ & \\
\hline $1997 \mathrm{RT}_{5}$ & QB1 & $21 / 07 / 2$ & $7.610 \pm$ & $12.345 \pm 6.149$ & $1.075 \pm 0.1$ & $0.474 \pm 0$ & \pm 0.091 \\
\hline $1998 \mathrm{KG}_{62}$ & QB1 & $21 / 07 / 2001$ & $931 \pm 0.104$ & $24.644 \pm 6.750$ & $1.123 \pm 0.131$ & $0.638 \pm 0.112$ & \pm 0.112 \\
\hline $1998 \mathrm{KR}_{65}$ & QB1 & average $^{(a)}$ & 81 & 092 & & & \\
\hline $1998 \mathrm{QM}_{107}$ & Cent & $21 / 07$ & $10.891 \pm 0.083$ & 7.769 & .100 & $0.474 \pm$ & $0.368 \pm 0.102$ \\
\hline $1999 \mathrm{HC}_{12}$ & QB1 & $7 / 0$ & 25 & 8.01 & & $0.490 \pm$ & 125 \\
\hline $1999 \mathrm{HX}_{11}$ & Plut & $25 / 05 / 2$ & $432 \pm 0.063$ & $12.410 \pm 4.280$ & $0.650 \pm 0.085$ & $0.527 \pm 0$ & $0.412 \pm 0.075$ \\
\hline $1999 \mathrm{KR}_{16}$ & QB1 & $27 / 04 / 2$ & $885 \pm 0.067$ & $48.685 \pm 3.378$ & $1.002 \pm 0$ & $0.826 \pm 0$ & .062 \\
\hline $1999 \mathrm{OM}_{4}$ & QB1 & $21 / 07$ & $.521 \pm 0.100$ & $20.038 \pm 5.954$ & $1.137 \pm 0.135$ & $0.602 \pm 0.104$ & $0.499 \pm$ \\
\hline $1999 \mathrm{OY}_{3}$ & QB1 & 1 & .056 & $5 \pm 4.059$ & 074 & $0.358=$ & 086 \\
\hline $1999 \mathrm{RB}_{216}$ & QB1 & $25 / 0^{-}$ & $7.668 \pm 0.087$ & $14.558 \pm 4.199$ & $0.897 \pm 0.122$ & $0.522 \pm 0.073$ & $0.506 \pm 0.061$ \\
\hline $1999 \mathrm{RD}_{215}$ & Scat & $15 / 08 / 2001$ & $7.863 \pm 0.079$ & - & - & - & $0.498 \pm 0.058$ \\
\hline $1999 \mathrm{RE}_{215}$ & QB1 & average $^{(b)}$ & $6.587 \pm 0.132$ & $30.747 \pm 4.969$ & $1.003 \pm 0.130$ & $0.697 \pm 0.070$ & $0.559 \pm 0.124$ \\
\hline $1999 \mathrm{RJ}_{215}$ & Scat & $22 / 07 / 2001$ & $7.881 \pm 0.103$ & $1.400 \pm 5.640$ & $0.919 \pm 0.144$ & $0.302 \pm 0.099$ & $1 \pm 0.081$ \\
\hline $1999 \mathrm{RY}_{215}$ & QB1 & $21 / 07 / 200$ & $7.430 \pm 0.077$ & $2.542 \pm 5.546$ & $0.645 \pm 0.092$ & $0.358 \pm 0.090$ & $0.453 \pm 0.096$ \\
\hline $1999 \mathrm{RZ}_{215}$ & Scat & $21 / 07 / 2001$ & $.072 \pm 0.079$ & $19.428 \pm 5.470$ & $0.771 \pm 0.097$ & $0.575 \pm 0.090$ & $0.539 \pm 0.091$ \\
\hline $2000 \mathrm{CL}_{104}$ & QB1 & $23 / 05 / 2001$ & $7.057 \pm 0.117$ & $29.523 \pm 5.600$ & $1.223 \pm 0.167$ & $0.628 \pm 0.095$ & $0.708 \pm 0.087$ \\
\hline $2000 \mathrm{~EB}_{173}$ & Plut & average $^{(c)}$ & $4.961 \pm 0.044$ & $25.362 \pm 2.711$ & $0.958 \pm 0.050$ & $0.646 \pm 0.044$ & $0.554 \pm 0.045$ \\
\hline $2000 \mathrm{EC}_{98}$ & Cent & $27 / 04 / 2001$ & $9.964 \pm 0.059$ & $9.058 \pm 3.425$ & $0.854 \pm 0.081$ & $0.466 \pm 0.050$ & $0.439 \pm 0.076$ \\
\hline $2000 \mathrm{EE}_{173}$ & Scat & $29 / 04 / 2001$ & $8.597 \pm 0.055$ & $13.453 \pm 3.092$ & $0.707 \pm 0.076$ & $0.496 \pm 0.046$ & $0.535 \pm 0.062$ \\
\hline $2000 \mathrm{FD}_{8}$ & QB1 & $29 / 05 / 2001$ & $7.034 \pm 0.104$ & $29.022 \pm 4.335$ & $1.121 \pm 0.153$ & $0.677 \pm 0.080$ & $0.594 \pm 0.053$ \\
\hline $2000 \mathrm{GN}_{171}$ & Plut & $27 / 04 / 2001$ & $6.187 \pm 0.045$ & $25.549 \pm 2.610$ & $0.924 \pm 0.059$ & $0.622 \pm 0.038$ & $0.617 \pm 0.054$ \\
\hline
\end{tabular}

(1) Dynamical class: QB1 = Cubewano, Plut = Plutino, Scat = scattered disk object, Cent = Centaur; (2) Epoch observed, "average" if more epochs were observed, averaging method according to Hainaut \& Delsanti (2002); ${ }^{(3)}$ M11 is the absolute $R$ magnitude (not corrected for phase effects); ${ }^{(4)} \mathrm{Grt}$ is the spectral gradient $\mathcal{S}(\% / 100 \mathrm{~nm})$; individual results for "averaged" objects are as follows: ${ }^{(a)} 1998 \mathrm{KR}{ }_{65}: 18 / 05 / 2001$ $B=25.482 \pm 0.074 R=23.662 \pm 0.067 ; 30 / 05 / 2001 B=24.149 \pm 0.060 \mathrm{~V}=23.192 \pm 0.051 R=22.669 \pm 0.024 I=21.847 \pm 0.032$; (b) $1999 \mathrm{RE}_{215}: 22 / 07 / 2001 B=24.356 \pm 0.075 \mathrm{~V}=23.431 \pm 0.057 R=22.735 \pm 0.030 I=22.273 \pm 0.039 ; 25 / 07 / 2001 B=24.741 \pm 0.087$ $V=23.651 \pm 0.061 R=22.954 \pm 0.045 I=22.271 \pm 0.045 ;^{(c)} 2000 \mathrm{~EB}_{173}: 19 / 04 / 2001 B=21.247 \pm 0.017 V=20.265 \pm 0.018 R=19.648 \pm 0.021$ $I=19.111 \pm 0.019 ; 27 / 04 / 2001 B=21.173 \pm 0.048 V=20.264 \pm 0.019 R=19.583 \pm 0.029 I=18.997 \pm 0.041$.

found. Here, we applied a slightly different approach to obtain realistic colors for this object in "conjugated" filters (for instance $V-R$ or $R-I$ ): since the filter exposures were not taken simultaneously, the brightness of the "conjugated" filter (for instance $R$ ) is estimated by linear interpolation of the filter brightness closest to the mid exposure time of the exposed filter (for instance $V$ or $I$ ). In this way, a small series of colors in "conjugated" filters is obtained and the mean values of the colors plus their standard deviations are calculated.

In addition to the classical aperture photometry we applied also the aperture correction method (Howell 1989) to a subsample of our data in order to compare the results obtained. Both methods gave the same results for the colors within the respective errors for the objects.

Error sources: the errors of the filter magnitudes are estimated as the sum of the absolute errors of the individual contributing parameters. Error contributions come from the basic data reduction (noise and flatness of sky background;
$1-2 \%)$, the uncertainty of the photometric calibration parameters (e.g. zero points, extinction, instrument colors; 3-8\%) and the measurement error of the actual object for the method used (1-5\%). In the case of SM with 1 to 2 standard star fields, the main error contributions are from the uncertainties of the zero points $(\sim 2 \%)$ and the errors of the (assumed) extinction parameters $(\sim 3-7 \%)$ when observations were made at high air masses. Error contributions in full aperture measurement result from variations in the sky background due to the relatively large aperture radius applied. Here, the impact of very faint extended sources in the apertures is not considered; however, the neighborhood of objects is carefully searched for indications of such "photometry contaminant's". The results from the aperture correction method are much less affected by sky noise (typically a factor of 2 less). Instead, error contributions can be due to variation's in the flat-field level, due to the companions of trailed object with stellar ones and due to effects from good seeing $\left(<0.6^{\prime \prime}\right)$ such as undersampling of the 


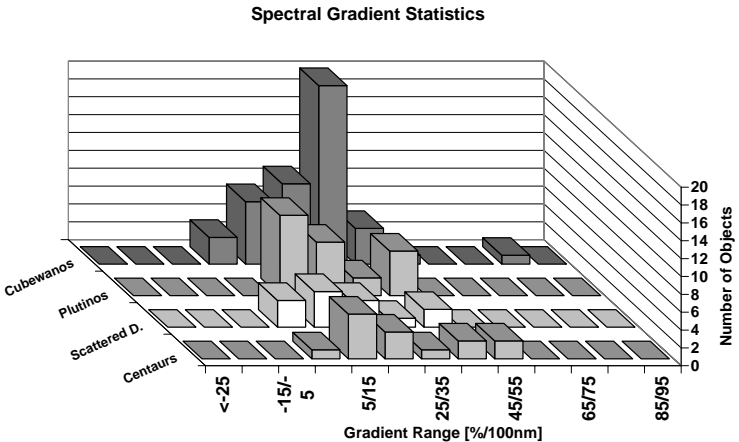

\begin{tabular}{|l|c|c|c|c|c|c|c|c|c|c|c|c|c|}
\cline { 2 - 13 } \multicolumn{1}{c|}{} & $<-25$ & $-25 /-15$ & $-15 /-5$ & $-5 /+5$ & $5 / 15$ & $15 / 25$ & $25 / 35$ & $35 / 45$ & $45 / 55$ & $55 / 65$ & $65 / 75$ & $75 / 85$ & $85 / 95$ \\
\hline Centaurs & 0 & 0 & 0 & 1 & 5 & 3 & 1 & 2 & 2 & 0 & 0 & 0 & 0 \\
Scattered D. & 0 & 0 & 0 & 3 & 4 & 3 & 1 & 2 & 0 & 0 & 0 & 0 & 0 \\
\hline Plutinos & 0 & 0 & 0 & 0 & 9 & 6 & 2 & 5 & 0 & 0 & 0 & 0 & 0 \\
\hline Cubewanos & 0 & 0 & 0 & 3 & 7 & 9 & 20 & 4 & 1 & 0 & 0 & 1 & 0 \\
\hline
\end{tabular}

Fig. 1. Spectral gradient histograms of Cubewanos, Plutinos, Centaurs and scattered disk objects. The histogram shows the distribution of the number of objects per spectral gradient interval for the various dynamical classes. The gradient intervals are given at the abscissa. The table at the bottom lists the numbers plotted in the histograms.

point-spread-function (FORS1 pixel size is $0.2^{\prime \prime}$ ) and image quality changes across the field of view due to the imaging optics of the instrument. Advantages and disadvantages of the aperture correction method are discussed in Howell (1989) and Barucci et al. (2000). The errors due to trailing, under-sampling and change of image quality $(<2 \%)$ are considered for the total error budget of this method.

The uncertainties of the color data (see Table 1) are calculated by formal quadratic error propagation.

\section{Photometry results}

The absolute brightness, spectral gradient and colors together with the dynamical type of the object are listed in Table 1 . The absolute brightness is calculated without phase angle correction (this correction is small - order of $\sim 0.05 \mathrm{mag}$ - since the phase angles do not exceed 2 deg in most cases). The spectral gradient is derived as described by Boehnhardt et al. (2001). If applicable, the results are averaged over the number of observed epochs. Reflectivity plots (not shown here) as presented in Delsanti et al. (2001) were used to verify the overall agreement of the slope of the individual color gradients with the overall trend over the $B-I$ wavelength range.

Overview: 18 of the 28 targets were measured for the first time, 10 objects have color data published by other groups. For 6 objects (1966 RR $20,1997 \mathrm{QH}_{4}, 1998 \mathrm{QJ}_{4}, 1998 \mathrm{KG}_{62}$, $1999 \mathrm{OY}_{3}, 2000 \mathrm{~EB}_{173}$ ) our results are in good agreement with those of various other groups (for comparison data see Hainaut \& Delsanti 2000). Deviations exist for 4 objects (1994 $\mathrm{EV}_{3}$, $1996 \mathrm{SZ}_{4}, 1998 \mathrm{QM}_{107}, 1999 \mathrm{KR}_{16}$ ).

$1994 \mathrm{EV}_{3}$ appeared as an outlier in the previous color studies (Luu \& Jewitt 1996; Boehnhardt et al. 2001; Gill-Hutton et al. 2001). The new observations of this object display brightness variations during our RBVIRBVI exposure series (with total execution time of $120 \mathrm{~min}$ ), namely for the $B$ and $V$ filters $(\sim 0.5 \mathrm{mag})$, that would result - if taken per se - in a spectrum of variable slope both over wavelength and in time.
However, if the measurements are used to calculate "quasisimultaneous" colors for "conjugated" filters as described in Sect. 3, this object appears to be a "normal" very red Cubewano with a rather uniform spectral slope. Hence, the outlier character of $1994 \mathrm{EV}_{3}$ may be due to significant brightness variability on a short time scale caused by cross-section and/or albedo variations over the rotation phase.

$1996 \mathrm{SZ}_{4}, 1998 \mathrm{QM}_{107}$ and $1999 \mathrm{KR}_{16}$ have deviations only slightly outside the error bars of the various published results. These differences could be due to intrinsic variability of the objects provided that no systematic errors exist between the results of the various groups. The latter, however, is not verified.

\section{Spectral properties of the dynamical classes of TNOs and Centaurs}

In this section we discuss trends in the color-color distribution of TNOs and Centaurs and we present the spectral gradient distributions of the various dynamical classes, i.e. for Cubewanos, Plutinos, scattered disk objects and Centaurs, as well as versus orbital properties of the objects. The goal of the latter exercise is to draw conclusions on specific physical characteristics that could be typical for the various object types and/or indicative of the influence of certain physical processes on their global surface properties. Three processes are proposed to affect the spectral gradients of TNOs and Centaurs: (1) high energy radiation aging, producing red colors and steeper gradients in the visible wavelength range (Strazzulla \& Johnson 1991; Shul'man 1972), (2) impact resurfacing producing gray colors through fresh ice deposits from the impact craters (Stern 1995) and (3) intrinsic activity acting in a similar way as impact resurfacing, i.e. causing gray colors through fresh ice deposits (Hainaut et al. 2000; Boehnhardt et al. 2001).

Data set: the data set for this discussion is based on spectral gradients of objects listed in the summary Tables 2 and 3 of Hainaut \& Delsanti (2002) supplemented by our new data as given in Table 1. For each object the averaged spectral gradient was calculated using all available data and following the procedure outlined in Hainaut \& Delsanti (2002). The merged database has spectral gradients of in total 95 objects of known dynamical type, of which 14 are Centaurs, 45 Cubewanos, 22 Plutinos and 13 scattered disk objects. One object (1994 JV) for which sufficient color data are available is lost and no dynamical type could be determined from the observed orbit arc. It is therefore excluded from the subsequent analysis.

\subsection{Color-color trends}

The objects display the normal range of TNO and Centaur colors known from other data sets, and follow the overall reddening trend of the objects (Boehnhardt et al. 2001; Delsanti et al. 2001; Trujillo et al. 2002). Following the statistical procedure described in Hainaut \& Delsanti (2002), the data subsets of the Plutinos and Cubewanos were tested for bimodality in the color-color distribution as reported (at least for Cubewanos) by Tegler \& Romanishin (1998, 2000): unfortunately, their conclusion does not appear statistically substantiated in our somewhat larger data set. However, a small clustering of red 
Spectral Gradient vs Excitation Parameter

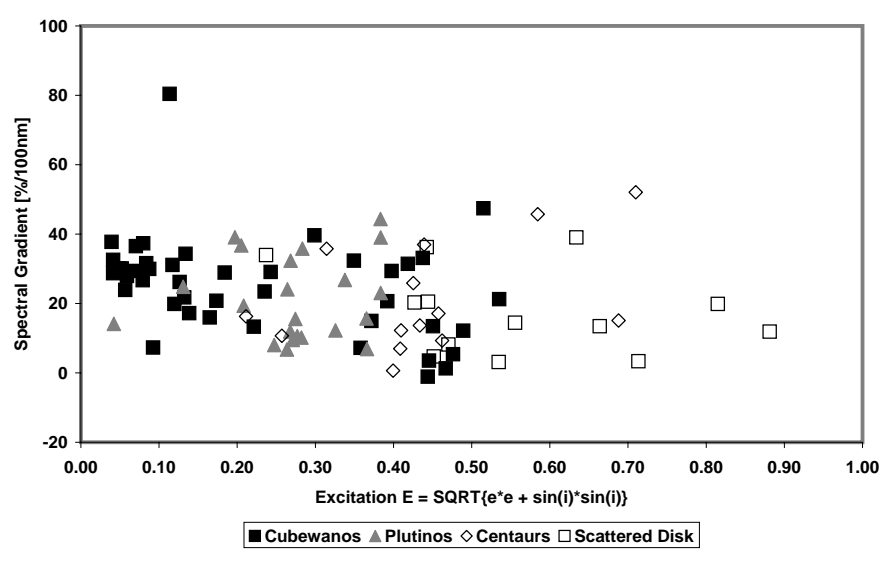

Fig. 2. Spectral gradient versus orbital excitation $E$. The plot shows the spectral gradient over the excitation parameter $E$ of the orbits for Cubewanos, Plutinos, Centaurs and scattered disk objects. The Cubewano and Plutino populations show upper limits in $E$ due to the absence of Neptune-crossing orbits with high eccentricity.

Cubewanos is noteworthy (see also Sect. 5.2). The $V-R$ versus $B-I$ trend suggests that the spectral slope in the $V-R$ region is a good indicator for the gradient over the larger $B-I$ wavelength range.

\subsection{Spectral gradient statistics}

Counting the number of objects per spectral gradient interval (defined by the average uncertainty value of $<10 \% / 100 \mathrm{~nm}$ of the spectral gradients in the database) for the 4 dynamical classes gives the histogram distributions in Fig. 1. With one exception (i.e. $1994 \mathrm{ES}_{2}$ with gradient $\sim 80 \% / 100 \mathrm{~nm}$ ) all objects have spectral gradients within -5 to $55 \% / 100 \mathrm{~nm}$. 5145 Pholus, previously also considered an "outlier" Centaur (52\%/100 nm), appears to be only at the tip of a "tail" of very red objects, since the Centaur 7066 Nessus $(45 \% / 100 \mathrm{~nm})$ and the Cubewano $1999 \mathrm{KR}_{16}(50 \% / 100 \mathrm{~nm})$ have reddening gradients not much smaller and thus they are starting to fill the gap in the previously published spectral gradient distributions of TNOs and Centaurs (Barucci et al. 2001; Boehnhardt et al. 2001).

The Cubewanos show a pronounced peak ( 20 objects) in the reddening range $25-35 \% / 100 \mathrm{~nm}$. The Plutinos have a maximum for 5-15\%/100 nm slope with the possibility of a second only very red population (peak at $35-45 \% / 100 \mathrm{~nm}$ ). The peak among the Centaurs at $5-15 \% / 100 \mathrm{~nm}$ seems to confirm earlier suggestions by Boehnhardt et al. (2001) and Delsanti et al. (2001). The scattered disk objects show a weak maximum around 5-15\%/100 nm. From Fig. 1 it appears that the gradient distribution of the Cubewanos differs from that of the Plutinos. The ones of the Centaurs and scattered disk objects have some similarity to that of the Plutinos, i.e. the peak at reddening of $5-15 \% / 100 \mathrm{~nm}$, even though the overall sample of the former object types is not yet large enough to consider it a firm conclusion.

\subsection{Spectral gradient and orbital parameters}

Here we analyse the spectral gradient distribution versus orbit excitation as indicator for the collision probability and severity and versus perihelion distance as tracer for effects related to intrinsic activity driven by Sun illumination.

Spectral gradients and orbit excitation parameter: various authors have analyzed the published color data of TNOs and Centaurs for correlations with orbital parameters. Most noteworthy is the conclusion by Trujillo et al. (2002), Tegler \& Romanishin (2000), and Hainaut \& Delsanti (2002) that Cubewanos appear redder for smaller eccentricity $e$ and inclination $i$. Both $e$ and $i$ contribute to the so called excitation parameter $E=\left(e^{2}+\sin ^{2} i\right)^{1 / 2}$ of the orbit, i.e. the object's velocity perpendicular to the Ecliptic and in radial direction. $E$ is an estimate for the velocity of the object with respect to another object at the same distance, but in a circular orbit and it might thus be related to the collision velocity and/or collision probability.

Figure 2 shows the distribution of spectral gradients versus excitation parameter $E$ for the objects of the four dynamical classes. Cubewanos with low excitation parameters appear to be of red color. Only two objects (1998 $\mathrm{SN}_{165}$ and $\left.1998 \mathrm{WV}_{24}\right)$ with low spectral gradient and low excitation parameter $E$ are found. The range of reddening increases with increasing excitation parameter $E$ until the maximum range $(\sim 0-50 \% / 100 \mathrm{~nm})$ is reached for excitations of $0.3-0.4$ or higher. This seems to be valid for all dynamical classes. The maximum reddening value may increase slightly with increasing $E$.

We also tested the relationship between spectral gradients and inclination (as proposed for Cubewanos and scattered disk objects by Trujillo \& Brown 2002) as well as between spectral gradient and eccentricity using linear regression fits to the data. In the case of the inclination there is a weak correlation (correlation coefficient $\sim 0.2)$ for Cubewanos $(\sim 0.65 \% / 100 \mathrm{~nm})$ and for scattered disk objects $(\sim 0.76 \% / 100 \mathrm{~nm} / \mathrm{deg})$. The trend of the reddening versus inclination is visualized in Fig. 3. However, the scatter in the spectral gradients is very large, and deviating from the conclusion by Trujillo \& Brown there might be "contamination" by a perihelion dependence in the data (see next paragraph). In the case of spectral gradients versus eccentricity, no correlation with the spectral gradients seems to exist for Cubewanos and scattered disk objects. For Plutinos and Centaurs correlations do not exist either with inclination nor with eccentricity (correlation coefficient $<0.05$ in all cases).

Spectral gradients versus perihelion distance: the diagrams in Fig. 4 plot the spectral gradient versus perihelion distance. The bubble diagrams visualize a third parameter via the size of the bubbles, i.e. the eccentricity (left panel) and the inclination (right panel) of the objects, respectively. The sub-panels show a very similar distribution of the objects, namely:

1. a cluster ( 9 out of 9 ) of red Cubewanos (spectral gradients $\sim 30-40 \% / 100 \mathrm{~nm}$ ) for perihelion distances above $41 \mathrm{AU}$. As demonstrated in the bubble diagrams, all objects of the red Cubewano cluster have orbits with low inclination AND low eccentricity (consequently also with low excitation parameter); 
Spectral Gradient vs Inclination - Comparison Measurements -- Fit

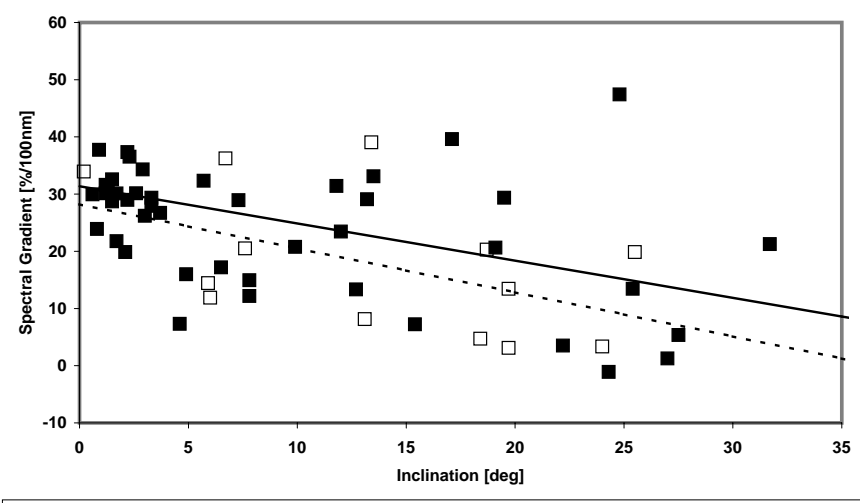

- Cubewano (measured) $\square$ Scattered Disk (measured) — Cubewano (fit) - - - Scattered Disk (fit)

Fig. 3. Trend between spectral reddening and inclination for Cubewanos and scattered disk objects. The figure shows the measured spectral gradient of Cubewanos and scattered disk objects versus the inclination of their orbits. The solid line shows the trend from a linear regression fit of the data for Cubewanos, the broken line the one for scattered disk objects. $1994 \mathrm{ES}_{2}$ (spectral gradient $\sim 80 \% / 100 \mathrm{~nm}$ ) is included in the fit, but not shown in this plot.

2. the reddening range of the objects increases with decreasing perihelion distance between $\sim 41$ to $\sim 36 \mathrm{AU}$ (from $\sim 30-40 \% / 100 \mathrm{~nm}$ to $\sim 0-50 \% / 100 \mathrm{~nm}$ ). This affects Cubewanos and scattered disk objects (the known Plutinos and Centaurs do not populate that perihelion distance range). The increase of the reddening range could be linear with decreasing perihelion distance (slope $\sim 3 \% / 100 \mathrm{~nm} / \mathrm{AU}$ beyond $35 \mathrm{AU}$, correlation factor of linear least square fit is $\sim 0.25$ );

3 . the reddening range remains constant $(\sim 0-50 \% / 100 \mathrm{~nm})$ for Centaurs, Plutinos, and Cubewanos with perihelion closer than 35 AU. Scattered disk objects are all below $20 \% / 100 \mathrm{~nm}$ when their perihelion is closer than $36 \mathrm{AU}$;

4. a small preference (12 out of 17) of spectral gradients $<20 \% / 100 \mathrm{~nm}$ may exist for objects with perihelion closer than $25 \mathrm{AU}$ from the Sun.

The cluster of red Cubewanos is confined to rather circular orbits in the Ecliptic. The absence of gray or less red Cubewanos with similar orbital parameters could indicate that the known resurfacing mechanisms that can produce gray colors in TNOs (impacts and intrinsic activity) are not very efficient for the cluster members. It would also imply that the surfaces of these objects are not exposed long or intense enough to space weathering to develop a gray color through radiation aging as proposed for TNOs based on laboratory work (Thompson et al. 1987).

The increase in the reddening range for decreasing perihelion distance is associated with many objects in orbits of higher excitation parameters and could potentially be explained by the scenario "radiation aging plus impact resurfacing". However, this scenario must also satisfy the perihelion dependence of the reddening and the existence of the cluster of red Cubewanos described above. A detailed modeling is needed to draw firm conclusions on the relevance of this scenario.
Using the picture of intrinsic activity for the explanation of the spectral gradient properties described above, we conclude that this activity should become important for resurfacing between 36-41 AU from the Sun. At this distance range the Sun illumination is still intense enough to sublimate CO and $\mathrm{N}_{2}$ ices of the surface (Delsemme 1982). One of the difficulties in this scenario is to explain the existence of red objects closer than $\sim 35$ AU from the Sun. Since cometary activity, if present, may be repetitive on much shorter time scales than radiation aging, the existence of red objects among Centaurs, Plutinos, Cubewanos and scattered disk objects with perihelia inside $\sim 41 \mathrm{AU}$ could imply that these objects are covered by an inactive surface crust. The trigger for intrinsic activity could be collision cratering when fresh and more active material is excavated and/or re-condensed to/on the surface. However, apart from Pluto no direct evidence for surface activity exists in TNOs and only indirect conclusions are published (Hainaut et al. 2000; Sekiguchi et al. 2002).

\section{Discussion}

During the first semester of our ESO Large Program on physical studies of TNOs and Centaurs we observe BVRI photometry of 28 objects of which 18 were measured for the first time. Our new observations confirm the results for the 10 objects for which color data are already published. Short-term (order of one hour) color changes are found for $1994 \mathrm{EV}_{3}$. Cross-section and/or intrinsic color variations over rotation phase could explain the observations.

Combination of our photometry results with the compilation of published colors (Hainaut \& Delsanti 2002) provides a new database of photometric spectral gradients of 95 objects. The bimodality character in the color-color distributions of the objects could not be verified. The gradient statistics of the Cubewanos is significantly different from those of the three other groups: a red population of Cubewanos (spectral gradients 30-40\%/100 nm) exists in rather circular orbits close to the Ecliptic with perihelion distances $>41$ AU. The reddening range of objects increases with decreasing perihelion distance and may also be correlated with the excitation parameter of their orbits. The weak correlations between spectral gradients and inclination for Cubewanos and scattered disk objects needs confirmation by observations of more objects.

The interpretation of the spectral gradient distributions and the relationship between physical and dynamical parameters of Cubewanos, Plutinos, Centaurs and scattered disk objects is unsolved. Radiation aging seems to cause the red population of Cubewanos beyond 41 AU from the Sun. However, the reason why some of the objects appear to be less red to even slightly bluish when closer to the Sun and/or in excited orbit is unclear and resurfacing by impacts or intrinsic activity are still viable scenarios. Certainly, modeling results addressing the reddening behavior of the various dynamical classes quantitatively for the different physical scenarios would be very instrumental in disentangling the physical and dynamical relationship of the icy bodies in the outer solar system. 

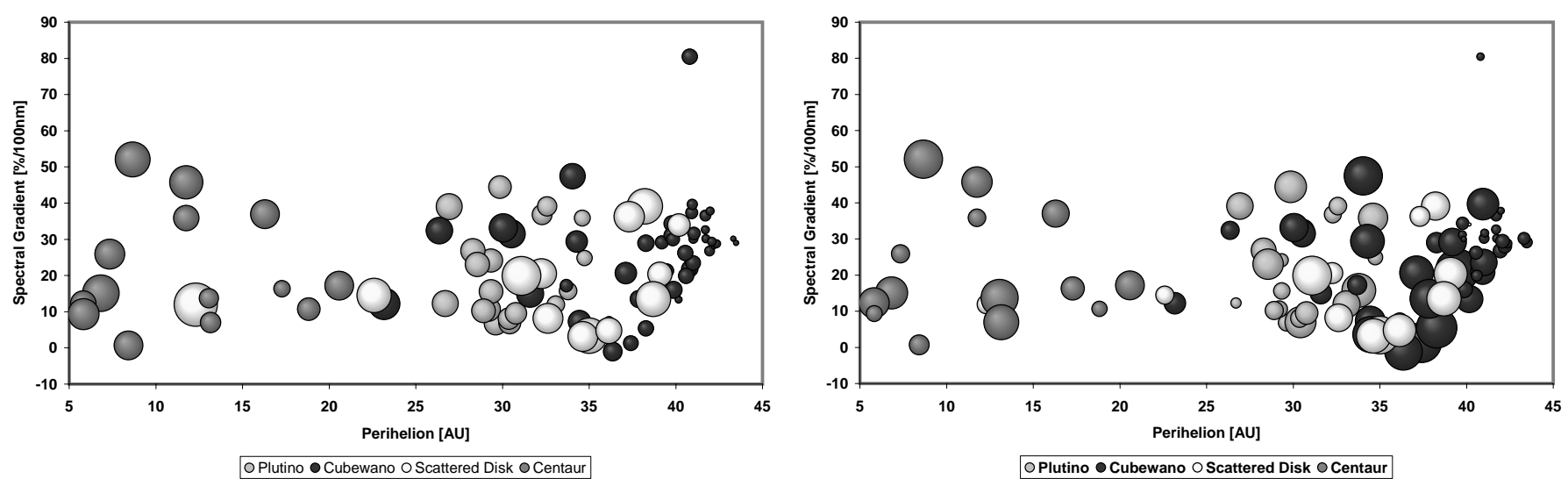

Fig. 4. Spectral gradient versus perihelion distance. The two bubble diagrams show the same distribution, using the size of the bubble as indicator for the eccentricity (left) and inclination (right) of the orbits.

Acknowledgements. We like to acknowledge the contributions of Dr. John Davies, Royal Observatory Edinburgh, and of Dr. Philippe Rousselot, Observatoire de Besancon, to our joint program. We thank very much the ESO Paranal Science Operations team (the night astronomers as well as the telescope and instrument operators having observed for our program, namely N. Ageorges, H. Alarcon, M.-T. Acevedo, V. Doublier, C. Herrera, N. Hurtado, E. Jehin, C. Lidman, A. Lopez, O. Marco, C. McKinstry, J. Navarrete, E. Pompei, R. Scarpa, A. Zarate) and the User Support Group ESO Garching for the excellent support and dedicated work devoted to the preparation and execution of this program for service mode observing at the VLT.

\section{References}

Barucci, M. A., Romon, J., Doressoundiram, A., \& Tholen, D. J. 2000, AJ, 120, 496

Barucci, M. A., Fulchignoni, M., Birlan, M., et al. 2001, A\&A, 371, 1150

Bessell, M. S. 1990, PASP, 102, 1181

Boehnhardt, H., Tozzi, G. P., Birkle, K., et al. 2001, A\&A, 378, 653

Boehnhardt, H., Barucci, M. A., Barrera, L., et al. 2002, SPIE, 4834, in press

Delsanti, A., Boehnhardt, H., Barrera, L., et al. 2001, A\&A, 380, 347

Delsemme, A. H. 1982, in Comets, ed. E. E. Wiikening (Univ. Arizona Press), 85
Doute, G., \& Schmitt, B. 1998, JGR, 103E, 31367

Gill-Hutton, R., \& Licandro, J. 2001, Icarus, 152, 246

Hainaut, O. R., Delahodde, C. E., Boehnhardt H., et al. 2000, A\&A, 356,1076

Hainaut, O. R., \& Delsanti, A. 2002, A\&A, 389, 641

Howell, S. B. 1989, PSAP, 101, 616

Landolt, A. U. 1992, AJ, 104, 340

Levison, H. F. 2002, IAU Joint Discussion 4, in Highlights in Astronomy, ed. H. Rickman, in press

Luu, J., \& Jewitt, D. 1996, AJ, 112, 2310

Sekiguchi, T., Boehnhardt, H., Hainaut, O. R., \& Delahodde, C. 2002, A\&A, 385, 281

Shul'man, L. M. 1972, in The Motion, Evolution of Orbits, and Origin of Comets, ed. G. A. Chebotarev, E. I. Kazimirchak-Polonskaia, \& B. G. Marsden (Reidel Dordrecht), IAU Symp., 45, 265

Strazzulla, G., \& Johnson, R. E. 1991, in Comets in the Post Halley Era, ed. R. E. Newburn, M. Neubegbauer, \& J. Rahe, 243

Stern, S. A. 1995, AJ, 110, 856

Tegler, S. C., \& Romanishin, W. 1998, Nature, 392, 49

Tegler, S. C., \& Romanishin, W. 2000, Nature, 407, 979

Thompson, W. R., Murray, B. G. J. P. T., Khare B. N., \& Sagan, C. 1987, JGR, 92, 14933

Trujillo, CA., \& Brown, M. E. 2002, AJ, 566, L125 